\title{
MUJER, FAMILIA Y AGRICULTURA Análisis de las perspectivas teóricas en Francia
}

\author{
PHILIPPE CARDON \\ Universidad de Franche-Comté. Francia
}

\author{
PALABRAS CLAVE ADICIONALES \\ ADDITIONAL KEYWORDS \\ Agricultura familiar, Francia, Género, Sociología Family Farm, France, Rural Sociology, \\ rural. \\ Gender.
}

\begin{abstract}
RESUMEN. El autor analiza la bibliografia sociológica y etnológica francesa sobre la mujer en la agricultura familiar desde los años 60. Destaca tres periodos distintos, en cada uno de los cuales domina una perspectiva teórica sobre la agrícultura familiar y la posición de la mujer en ella. Si en los años 60-70 predominaba una concepción funcionalista de la familia agrícola (vínculos familiares orgánicos, complementaridad hombre/mujer), emerge en los años 90 una concepción individualista en la que están presentes los vínculos familiares relacionales y una identidad individualizada de la mujer y del hombre. En la segunda parte del artículo, el autor propone una nueva perspectiva de análisis de la mujer en la agricultura familiar, haciendo hincapié en un tema tan importante como es la transmisión patrimonial.
\end{abstract}

ABSTRACT. The author presents a critical analysis of french sociological and ethnological literature about women and the family agriculture since the 60 's. Firstly, he describes three different periods of time and for each one presents a theoretical conception. In the 60's and 70's a fonctionalist conception of the family agriculture predominated (" organic "family, men and women complementarity). In the 90 's an individualistic conception of the family emerged, based upon family relational ties and distinctive identity of women and men. Secondly, the autor presents a new approach to the relationship between women and the agricultural family focussing on the handing down of patrimony within the family agriculture.

En Francia, desde los años 60, la sociología y la antropología se han ocupado extensamente de la cuestión de la mujer en la agricultura familiar. Sin duda, se refleja en ello la aparición de una importante literatura sociológica sobre las mujeres en aquella época.

E-mail: philippecardon@voila.com

Revista Internacional de Sociología (RIS)

Tercera Época, nº 29, Mayo-Agosto, 2001, pp. 191-207. 
RIS

Al margen de lo excepcional de la abundante literatura científica sobre ese tema, nos parece pertinente examinar con atención los contenidos y las perspectivas teóricas que en ella emergen. A partir de una serie de libros y estudios fundamentales que han marcado la sociología y etnología francesa, nos interrogaremos sobre cómo etnólogos y sociólogos han analizado el vínculo entre mujer y agricultura familiar.

La hipótesis general es que en 40 años hemos pasado de una concepción funcionalista a una concepción individualista de la familia. En ese periodo encontramos, sobre todo, una problemática centrada en la identidad y la cuestión del estatuto social y profesional del cónyuge del agricultor.

La primera parte del artículo presenta una síntesis de las distintas perspectivas teóricas sobre la mujer en la agricultura en tres fases históricas. Puesto que el 90\% de las explotaciones agrícolas francesas se transmiten de padre a hijo', podemos decir que la agricultura sigue siendo familiar (aunque el vínculo familia/trabajo ha cambiado). Y con mucha razón las investigaciones sobre las mujeres en el medio rural siempre han analizado la cuestión de su identidad respecto a la familia. Dar cuenta de esas perspectivas teóricas sobre la mujer y proponer una crítica no puede hacerse prescindiendo de una reflexión sobre la familia.

En la segunda parte, proponemos una síntesis crítica de estas perspectivas hacia una nueva problemática sobre la mujer en la agricultura al hacer hincapié en un tema tan crucial como es la transmisión patrimonial.

\section{EL DIFÍCIL RECONOCIMIENTO PROFESIONAL DE LAS MUJERES EN LA AGRICULTURA}

En esta primera parte veremos cómo sociólogos y etnólogos han analizado la identidad de las mujeres respecto a la agricultura familiar, tomando como indicadores los elementos siguientes: los vínculos familiares, los vínculos de género, los vínculos mujer/trabajo agrícola y los vínculos mujer/patrimonio en la agricultura.

A partir de este esquema de análisis, podemos reagrupar los distintos trabajos sobre las mujeres en la agricultura en tres periodos históricos en función de la evolución de la familia (la entrada de la familia en un periodo de mutaciones sin precedentes desde los años '60) y de la evolución de la sociología especializada en un terreno que se ha desarrollado considerablemente desde esa época.

\footnotetext{
${ }^{1}$ Coloquio Transmission des exploitations agricoles et installation des agriculteurs dans la communauté europénne, 10-11 décembre 1993, INRA, Dijon.
} 


\section{La época posterior a la II Guerra Mundial}

Después de la Segunda Guerra Mundial, la agricultura europea experimenta una mutación extraordinaria y entra en un proceso de modernización, necesario para su integración en una economía de mercado. En Francia, en ese contexto de cambio económico y social, etnólogos y sociólogos rurales intentan describir las transformaciones de la agricultura. Sus distintos estudios, generalmente monográficos, son de dos tipos:

a. Las investigaciones etnológicas de ese periodo analizan las sociedades rurales y la conservación del mundo rural frente a la modernización en marcha. En particular se interesaban por los vínculos entre sistemas de parentesco, organización familiar y prácticas sucesorias, con el fin de ver cuál era el papel de la herencia en los modos de conservación y transformación de las explotaciones familiares agrarias. Es el caso de Pierre Bourdieu (1972: 1105-1129) que analizó en este sentido los mecanismos de reproducción de las familias campesinas a través del vínculo entre modo de herencia, necesidad económica y mantenimiento del patrimonio. Por su parte, Françoise Zonabend (1980) mostró el papel que juega la parentela cercana o lejana en las estrategias matrimoniales. También Claude Karnoouh (1980) mostró cómo las estrategias matrimoniales tienen por objetivo ampliar las solidaridades de parentesco para favorecer el poder local. Georges Augustins, en su estudio comparativo a nivel europeo (Augustins, 1989), analizó los distintos modos de reproducción de los grupos domésticos en Europa y demostró la existencia de dos sistemas familiares distintos: la casa y la parentela. En general observamos cómo los etnólogos se interrogaban sobre la estructura del parentesco, el ciclo de la vida familiar y la transmisión del patrimonio.

b. Los sociólogos especializados en el mundo rural analizaron el impacto del capitalismo y la integración progresiva del modelo urbano en agricultura familiar con el paso de la sociedad campesina a la sociedad moderna. Es el caso del ya famoso libro de Henri Mendras La fin des paysans (Mendras, 1992) y de su tesis sobre la desaparición de las sociedades rurales y la aparición de agricultores-productores sometidos a las reglas del mercado y de la tecnología. En esa perspectiva, Jean-Pierre Darré (1985) analizó también la formación del grupo profesional de los agricultores, que se independizaba de la comunidad local. Marie Michel y Jean Viard (1977) analizaron el impacto del modelo urbano en cuatro pueblos rurales del sur de Francia.

El proceso de modernización y la urbanización de las sociedades rurales implicaba que la familia rural tradicional ampliada desaparecía en beneficio de una familia agrícola centrada en la pareja y ya no en el grupo de parentesco. En este sentido, el medio rural experimentaría el mismo proceso de individualización 
de la familia que el medio urbano.

Ese análisis se acercaba a la perspectiva teórica de Talcott Parsons sobre las sociedades industriales: el proceso de industrialización y modernización de la sociedad conduce a una segmentación de la familia mediante la reducción del grupo doméstico a la pareja, que se convierte en una unidad de residencia y consumo.

En esas perspectivas etnológicas y sociológicas se analizaba a la mujer integrada en ese todo inseparable de familia y agricultura, en el cual ella debía asumir papeles particulares (niños, esfera doméstica, ayuda al marido, etc.). Se observan dos temas recurrentes en las diferentes investigaciones:

En primer lugar, la división sexual del trabajo, que implica la existencia de una dicotomía entre la esfera de producción (masculina) y la esfera de reproducción (femenina). Es, por ejemplo, el caso de S.C. Rogers, que analiza los procesos de conservación del papel y lugar de la mujer en el medio rural. Yvonne Verdier (1979) describe los espacios típicamente femeninos no sólo a nivel de familia, sino también a nivel de la comunidad (pueblo, villa, ...). Esa división sexual del trabajo estaría siendo reforzada por el proceso de modernización de la agricultura en marcha desde los años 50, caracterizado entre otras cosas por la mecanización, un proceso que tendría como consecuencia el alejamiento de las mujeres de la esfera productiva y el reforzamiento de su papel doméstico (Filippi y Nicourt, 1981).

En segundo lugar, la importancia de la unidad conyugal como espacio de solidaridad y complementariedad entre hombre y mujer y cuyo objetivo era el equilibrio de la familia. Es la tesis defendida por Martine Segalen (1980), en particular respecto a la autoridad, al considerar que la relación marido-mujer se basa no en la autoridad absoluta de uno sobre otro, sino en la complementariedad entre los dos. Henri Mendras, por su parte, subrayaba esa fuerte imbricación entre familia y explotación agrícola y la complementariedad hombre-mujer: "Cada uno de los miembros de la familia es, al mismo tiempo, un compañero de trabajo: el padre conserva generalmente la gestión de la explotación, o bien permanece como consejero al que se respeta o al que se soporta; la mujer se ocupa de las actividades que le son propias, al tiempo que participa en numerosos trabajos propios de los hombres" (Mendras, op. cit.: 117). Esa complementariedad hombre/mujer favorecería así el vínculo fundamental entre la esfera de producción y la esfera de reproducción.

De esta forma, la familia agrícola, en la cual la mujer asumía las funciones de reproducción, tenía como función el mantenimiento de modelos culturales estables que favorecían la reproducción de los valores y sistemas simbólicos y la continuidad del grupo doméstico. Ese modelo de integración permitía la estabilidad del grupo doméstico. Y era la división sexual del trabajo la que garantizaba el equilibrio y la estabilidad del grupo, tal y como sostenía Talcott Parsons en su análisis funcionalista de la familia (Parsons y Bales, 1955). 
En efecto, el funcionalismo considera que los elementos que constituyen la sociedad forman un todo indisociable. La familia representa así un sistema que tiene como función asumir la construcción de las identidades de los hijos y la estabilidad de los padres. División sexual del trabajo y reparto de las tareas garantizan el equilibrio y la estabilidad de la familia que, con la industrialización y la modernización de la sociedad, tendería a centrarse en el núcleo familiar padres-hijos; de este modo, la familia conyugal se independiza del grupo de parentesco.

Eso es precisamente lo que reivindican, a partir de los años 60 en Francia, políticos y sindicatos, es decir una agricultura oficio de pareja donde se exprese la unidad familiar como repuesta necesaria a la evolución de un mundo agrícola obligado a entrar en el mundo moderno. Objeto de un intenso trabajo de construcción política e ideológica, esa agricultura oficio de pareja tenía que ser viable y permitir a una familia subsistir sin recurrir al trabajo exterior.

Aplicada a la agricultura, esa concepción funcionalista de la familia consideraba que la identidad de las mujeres (que asumen roles sexualmente definidos) es complementaria a la de los hombres. Dicha concepción parte de la base de que existen vínculos familiares, definidos como complementarios unos de otros, que actuarían como garantía de la estabilidad familiar.

Esa concepción familiarista de la agricultura se veía reforzada por una visión jurídica de las relaciones de trabajo como si fueran relaciones armoniosas, equilibradas y orientadas a un interés común (el patrimonio) que trasciende los intereses individuales (Lagrave y Caniou, 1987: 124). En efecto, en Francia, la agricultura no se rige por las leyes que rigen al mundo de la empresa, lo que conduce a una confusión jurídica entre la función productiva (comercial) del trabajo agrícola y la función patrimonial, es decir una confusión entre capital y patrimonio. Además, la agricultura no se rige por el Derecho del Trabajo, sino por el Derecho de la Familia y el de los regímenes matrimoniales, que son los que rigen las relaciones de trabajo y de status, "legislación que excluye todo tipo de vínculo contractual entre los miembros del grupo de trabajo" (idem, 123). De hecho, la mujer era en ese periodo, según Rose-Marie Lagrave y Juliette Caniou, una menor juridica, puesto que el marido era el único jefe de la explotación agrícola, siendo el estatuto de la mujer un estatuto dependiente de la familia.

\section{Los años 70-80 o los años feministas}

Los años 70-80 marcan un cambio radical. A lo largo de ese periodo de fuerte reivindicación social de las mujeres respecto a su estatuto social y profesional surge la cuestión de la profesionalización de las mujeres en la agricultura. En ese contexto aparecen nuevas reivindicaciones femeninas, que implican nuevas formas de relaciones de pareja y relativizan el modelo, demasiado perfecto, de 
la complementariedad hombre/mujer en la agricultura. Henri Mendras, en su capítulo "Postface . 20 ans après", de la reedición en 1984 de su libro La fin des paysans revisa lo que planteaba 20 años antes sobre el vínculo entre familia y explotación agrícola: "El capítulo III es, sin duda, hoy en día el más superado por la evolución de la sociedad y por los progresos de la investigación. Todo el análisis trata de la familia y de la explotación como un todo inseparable (...). Sin embargo, ahora hay que distinguir claramente entre familia y explotación agrícola, darle un giro a la perspectiva y abordarla desde el punto de vista de la familia" (Mendras, op.cit.: 377).

A partir de los años 70, sociólogos franceses intentaron analizar el grado de implicación de las mujeres en el trabajo agrícola y doméstico. El objetivo de esos trabajos empíricos era determinar la contribución de la mano de obra femenina en el trabajo agrícola, así como las formas de la división del trabajo y el reparto de los papeles entre marido y mujer. Así, subrayaban la fuerte imbricación, en el caso de las mujeres, entre trabajo doméstico y trabajo en la explotación agrícola.

En ese contexto surgen algunas investigaciones con un carácter crítico a nivel teórico y que vuelven a cuestionar el modelo de la agricultura como un oficio de pareja. Alice Barthez (1992) muestra así la naturaleza contradictoria de la explotación agrícola. En efecto, señalaban que si bien la explotación agrícola se ha convertido en una empresa integrada en el mercado, al mismo tiempo ha conservado un funcionamiento familiar del trabajo. Rechazando fundamentalmente la visión familiarista de una agricultura basada en la complementariedad entre hombre y mujer, subrayaban la necesidad de analizar la familia agrícola, ya no como una unidad familiar, sino como un espacio de individuos coexistentes y las relaciones de trabajo como el reflejo de las relaciones jerárquicas de poder entre sexos y generaciones. En efecto, lo que caracteriza la organización del trabajo en la agricultura familiar es la gratuidad del trabajo de las ayudas familiares (esposa, niños), unos sujetos subordinados a un jefe de explotación que detenta el poder sobre el patrimonio agrícola, sobre el trabajo y sobre los medios de producción. De hecho, "el carácter productivo o improductivo del trabajo no depende tanto de su carácter intrínseco, como del estatuto de la persona que lo asume y realiza" (Barthez, 1992: 30).

En esa perspectiva, Martine Berlan (1985) desarrolla la tesis de la adaptabilidad permanente de las mujeres a las exigencias de la explotación agrícola. El trabajo de la mujer se caracteriza por su permanente disponibilidad: la mujer tiene que intervenir puntualmente en función de las necesidades de la explotación y de la familia. Esto es lo que conduce a Christian Nicourt y a Geneviève Filippi a considerar que el trabajo de las mujeres en la agricultura se presenta bajo la forma de secuencias inconclusas que "se interrumpen por la interacción con otros operadores o a raíz de acontecimientos sobrevenidos vinculados a lo aleatorio del trabajo agrícola: estado sanitario de la cabaña ganadera, cambio 
meteorológico..." (Nicourt, 1987: 478-479).

Esas distintas perspectivas llegan a una crítica de la idea de trabajo familiar y de la teoría de la complementariedad de los roles sexuales, en la misma línea que otros autores no franceses como Sarah J. Whatmore en Inglaterra ${ }^{2}$. Esta autora critica la perspectiva del ciclo familiar asociada a la teoría de los roles sexuales que considera que es la cantidad de responsabilidades domésticas asumidas por la mujer, la que determina el tipo de participación de aquélla en el trabajo agrícola. Para Whatmore, esa tesis oculta el carácter de subordinación del trabajo de la mujer. Según la tesis de esta autora, hay que buscar el origen de esa subordinación en los modos de transmisión del patrimonio agrícola y de los tipos de relaciones que supone. "La posición de la mujer en la estructura de parentesco de la explotación familiar viene definida por el matrimonio y por su papel de esposa, mientras que la posición del hombre aparece definida fundamentalmente por la herencia y por su condición de hijo" (Whatmore, 1989). La identidad social de la mujer se construye en relación a su estado matrimonial.

Paralelamente a esos trabajos empíricos, otros estudios hacen una crítica de las estadísticas, en particular de los censos agrarios. En efecto, las estadísticas no tienen en cuenta una serie de trabajos, en particular agrícolas, realizados por las mujeres, lo que tiende a reforzar no solamente la división esfera de producción masculinalesfera de reproducción femenina, sino también la dificultad para definir la categoría social de la agricultoras, cónyuges de agricultores (Lagrave, 1987).

Además, otras instituciones favorecen la reproducción de las desigualdades de género en el seno de la familia. Los sociólogos intentan comprender por qué mecanismos sociales el carácter gratuito del trabajo de las mujeres permanece invisible para ellas. De esta forma, no sólo la familia transmite una visión positiva de la división sexual del trabajo, sino también del aprendizaje agrícola femenino, tal y como lo demostraba Juliette Caniou (1980: 316), o lo planteaban los sindicatos (Martine Berlan y Rose-Marie Lagrave, 1985).

Así, los años 70-80 se caracterizan por una crítica radical de la concepción organicista de la agricultura familiar como un oficio de pareja y de la idea de complementariedad hombre/mujer. Son el carácter subordinado de la posición de la mujer y el dificil reconocimiento jurídico de un estatuto profesional para el cónyuge del agricultor lo que se subraya en estos trabajos. Todos los autores demuestran el carácter de dominación que suponen las relaciones de pareja. Esa dominación se expresa a través de la invisibilidad del trabajo de la mujer, de las

\footnotetext{
${ }^{2}$ El artículo de Whatmore (1989) es una sintesis de su tesis doctoral, The other half of the family farm (1988).
} 
RIS

REVISTA NTERNACIONAL DE SOCIOLOGIA

№ 29, Mayo - Agosto, 2001

PHILIPPE CARDON

relaciones de tipo patriarcal que construyen la familia agrícola y de la ausencia de un estatuto social y profesional del cónyuge del agricultor. Ese hecho se ve reforzado, tal y como ya lo hemos subrayado, por la aplicación del código civil a la agricultura, que tiene como consecuencia que, a efectos jurídicos, ser agricultora no es una profesión, sino un estado matrimonial, aunque, en el caso de Francia, por ejemplo, la ley de Orientación Agrícola del 4 de julio de 1980 reconoce a los dos cónyuges como co-titulares de la explotación, o la ley del 11 de julio de 1985 sobre los EARL (explotación agrícola de responsabilidad limitada) permite a una agricultora ser titular de una parte del capital, al igual que su marido. Pero esa evolución jurídica, en el caso de Francia, se refiere a la gestión de los bienes patrimoniales y no a los derechos profesionales de las mujeres. Todavía es la misma lógica patrimonial/matrimonial la que regula la profesión de las agricultoras. La ley sigue regulando las relaciones de las personas con los bienes, pero no las relaciones de trabajo entre las personas.

Durante ese periodo, los sociólogos, a través de sus críticas de la idea de complementariedad hombre-mujer, denuncian el no reconocimiento de la mujer del agricultor como sujeto. Desde esa perspectiva la identidad social y profesional de la mujer en la agricultura se construye en una relación de dominación y de subordinación a la de los hombres, dominación que se expresa en relaciones familiares patriarcales caracterizadas por la invisibilidad del trabajo femenino y la ausencia de un estatuto social y profesional del cónyuge del agricultor. Estos estudios se inscriben en el proyecto intelectual y científico del feminisno de aquella época, que, por una parte, denuncia el carácter gratuito del trabajo doméstico de la mujer fundado sobre la explotación patriarcal (Delphy, 1970) y, por otra, intenta constituir a la mujer en sujeto histórico: "Durante los años 1972-1980, lo que estaba en juego en los estudios feministas era fundamentar teóricamente las relaciones entre trabajo doméstico y trabajo asalariado, para que apareciera la parte de trabajo femenino invisible y no renumerado. El caso de las agricultoras ha sido el ejemplo que ha servido para desconstruir y reconstruir los lazos invisibles entre trabajo productivo y trabajo considerado improductivo" (Lagrave, 1998: 23).

\section{Los años 90: ¿hacia una individualización profesional?}

Ante el hecho de que el número de mujeres de agricultores que tienen una actividad asalariada fuera de la agricultura había aumentado a lo largo de los años 80 , los sociólogos tuvieron que pronunciarse sobre la existencia de nuevas formas de pareja y de familia agrícola. Frente al modelo ideal de la agricultura familiar como un oficio de pareja de los años 60-70, los años 80-90 ven emerger nuevas formas de parejas agrícolas en las cuales cada vez más mujeres parecían independizarse profesionalmente fuera de la agricultura.

En ese contexto, las investigaciones sobre el sector agrario consideran que 
esas nuevas formas de agricultura familiar (parejas en las que la esposa tiene un trabajo asalariado no agrícola por ejemplo) corresponden a la nueva forma de la familia contemporánea tal y como lo ha descrito François de Singly. Esa familia conyugal se caracteriza por su autonomía, que conduce a una disminución de los vínculos de parentesco, a la primacía de la pareja, y a la sicologización de las relaciones de pareja, llegando a la desaparición progresiva de la familia como institución. La familia vuelve a ser relacional puesto que privilegia lo afectivo y la autonomía de sus miembros. La tesis central del autor es que la familia contemporánea "se encuentra en el centro de la construcción de la identidad individualizada" (Singly, 1996: 36). La pareja valoraría entonces las relaciones basadas en una igualdad entre cónyuges que reivindican su autonomía, entre otros ámbitos, a nivel profesional.

La aplicación de ese modelo a la agricultura conduce a algunos autores a considerar que distintos modelos de familia agrícola coexistirían hoy, como sostiene Isabelle Van de Walle ${ }^{3}$ : a la familia tradicional patriarcal, caracterizada por un modo de organización familiar, en el cual predominan los valores y los comportamientos de la sociedad agrícola local, se opondría una familia moderna que privilegia la autonomía de los cónyuges y la valorización de dos proyectos profesionales distintos. Ese modelo familiar rompe con las concepciones dominantes de las relaciones familiares en la agricultura tradicional. Entre los dos se encontraría una familia donde lo conyugal, en las actividades privadas, trasciende los intereses individuales, a pesar de la individualización profesional de sus miembros.

En esa perspectiva, la oposición entre esos distintos modelos familiares se construye en la oposición entre patriarcado e igualdad, tradición y modernidad. Al modelo tradicional patriarcal de la familia se opondría un modelo moderno igualitarista. El trabajo asalariado aparece entonces, en el estado actual de las relaciones hombre/mujer, como la única manera, para las mujeres, de emanciparse de la tutela familiar tan presente en la agricultura.

Al mismo tiempo, la evolución jurídica del estatuto de las mujeres en la agricultura, y el desarrollo en Francia de nuevas actividades asumidas por las mujeres (camping, albergue, actividades de producción: fresas, etc.), permiten pensar en una nueva forma de reconocimiento profesional de las mujeres en el seno de las explotaciones agrarias (alejada del espacio de producción), tal y como lo reivindican los sindicatos que valoran "nuevos" proyectos profesionales

\footnotetext{
${ }^{3}$ Van De Walle, I. (1993), Terres des hommes, salaire des femmes. Stratégies individuelles et familiales en agriculture. Le cas du Calvados, thèse de $3^{\text {eme }}$ cycle (no publicada), bajo la dirección de Bertrand Hervieu.
} 
agrícolas elegidos por las mujeres. Después del "se vuelve uno agricultor por elección" (y no por obligación) de los años 80 (reivindicación de los hijos herederos), se pasaría a la reivindicación feminista de que "una se vuelve agricultora por elección" en los años 90 .

Esa idea se revela ampliamente en Francia a través de los medios de comunicación, que multiplican reportajes y debates sobre "las nuevas agricultoras", que reivindican haber elegido su oficio por vocación. Valoran una nueva agricultura que encarna modernidad, distinción, dominio de su porvenir, es decir una mujer "como las demás"4.

Así, las evoluciones sucesivas del sector agrario (modernización, evolución de la profesión, ...) y la evolución de la familia permiten pensar en la desaparición de la familia agrícola tradicional, la profesionalización de las mujeres fuera de la agricultura, $o$ a través de nuevas actividades en la explotación, tendiendo a confirmar ese proceso. Modernización de la agricultura y profesionalización de las mujeres confirmarían así la desaparición de la explotación agrícola tradicional y la aparición de nuevos vínculos familiares relacionales.

\section{MUJER, PARENTESCO Y PATRIMONIO}

¿Cuáles son las lecciones que se pueden sacar de la lectura de los estudios sobre la mujer en la agricultura francesa? Podemos destacar dos tendencias.

Por una parte, si bien en un principio fueron la etnología y la sociología rural las que se interesaron por la agricultura (analizando la familia agrícola como un todo en el cual cada uno asume papeles particulares), ha sido a través de una reflexión sobre el trabajo y el vínculo entre producción y reproducción como los estudios feministas han facilitado una crítica radical de la concepción orgánica, funcionalista, de la familia. Estos estudios denuncian el carácter gratuito e invisible del trabajo de las mujeres y la ausencia de un reconocimiento profesional de las mujeres en la agricultura. Trabajo, organización del trabajo y estatuto social, son los temas centrales de estos estudios.

Más tarde, será a través de una sociología basada en el individualismo metodológico que se analizará el papel de la mujer en la agricultura. La aplicación del modelo de la individualización y autonomía de la pareja y de sus miembros conduce a considerar que en el estado actual de las relaciones

\footnotetext{
${ }^{4}$ Ver el programa de televisión francesa del 5 de mayo de 1999 Ça se discute titulado Paysannes: le bonheur est-il dans le pré?, Antenne 2; el programa de televisión francesa del 3 de marzo de 2000 Côté week-end, côté cinquième titulado Les nouvelles agricultrices, La Cinquième, o el programa de radio del 9 de septiembre de 1999 Les agricultrices aujourd'hui, France Inter.
} 
hombre/mujer en la sociedad, sólo el trabajo asalariado externo permite a las mujeres emanciparse de la tutela familiar en la agricultura y tener un reconocimiento social y profesional.

Al final de estos análisis, que pretendían dar cuenta de la evolución de las perspectivas teóricas relativas a la cuestión de las mujeres y de la familia en la agricultura, se puede afirmar que las investigaciones sobre la mujer en la agricultura se enfrentan a la alternativa siguiente:

- O se interrogan sobre las agricultoras y la evolución de su estatuto en el seno de la explotación agrícola, analizando sus reivindicaciones y los cambios jurídicos de su estatuto, mostrando la complejidad del vínculo entre esfera doméstica y esfera profesional y su difícil reconocimiento profesional, reforzado por un trabajo ideológico tanto a nivel de la profesión como de las taxonomías estadísticas (Barthez, 1982; Lagrave y Caniou, 1987; Berlan, 1985 y 1987);

- o se interrogan sobre las mujeres asalariadas fuera de la agricultura, considerando el trabajo asalariado, bien como una necesidad económica o bien como una voluntad de la mujer de hacerse autónoma, apareciendo entonces para las mujeres como la única alternativa posible para escapar a la tutela familiar.

$\mathrm{El}$ análisis de la identidad de las mujeres en la agricultura se resume en la división entre el reconocimiento profesional exterior y el difícil reconocimiento profesional agrícola; solamente las actividades anejas a la explotación aparecen como nuevas formas de reconocimiento profesional de las mujeres en la agricultura.

Es, por tanto, un análisis en términos de trabajo, organización del trabajo y estatuto social lo que sirve para analizar la relación entre mujer y agricultura, en una problemática general de las relaciones de género. Ese doble análisis (sociología del trabajo y sociología de género) permite una crítica de una visión funcionalista de la familia. A la concepción orgánica y funcionalista de los sociólogos rurales y de los etnólogos de los años 60-70 se opondría una familia agrícola contemporánea donde mujer y marido construyen su propio porvenir profesional.

Se pueden resumir las evoluciones teóricas del análisis del vínculo entre mujer/familia/agricultura en la tabla 1. Naturalmente, esta tabla es una simplificación y no pretende agotar el conjunto de estudios. Probablemente, ciertos elementos que hubieran merecido ser integrados, han sido olvidados. Sin embargo, la sencillez de esta tabla es su virtud esencial: sirve de guía de lectura y permite dar cuenta de los debates teóricos en juego en la problemática de la identidad de las mujeres en el medio rural en Francia.

Lo que hoy está en juego es la reivindicación de una nueva imagen de la mujer en la agricultura. Ya no es esa mujer sometida a la explotación y al sistema patriarcal, sino una persona que ha elegido su destino: bien quedándose en la explotación agrícola y asumiendo nuevas tareas, o bien teniendo una actividad profesional exterior. 
RIS

REVISTA NTERNACIONAL DE SOCIOLOGIA

№ 29, Mayo - Agosto, 2001

PHILIPPE CARDON

Tabla 1.

\begin{tabular}{lccc}
\hline & Años 50-70 & Años 70-80 & Años 90 \\
\hline $\begin{array}{l}\text { Concepción } \\
\text { Teórica }\end{array}$ & Funcionalismo & $\begin{array}{c}\text { Feminismo } \\
\text { Existencialismo }\end{array}$ & Individualismo \\
$\begin{array}{l}\text { Vínculo } \\
\text { familiar }\end{array}$ & $\begin{array}{c}\text { Solidaridad } \\
\text { orgánica }\end{array}$ & $\begin{array}{c}\text { Explotación } \\
\text { patriarcal }\end{array}$ & $\begin{array}{c}\text { Vínculo } \\
\text { relacional }\end{array}$ \\
$\begin{array}{l}\text { Vínculo } \\
\text { hombre/mujer }\end{array}$ & Complementariedad & $\begin{array}{c}\text { Autoridad } \\
\text { dominación masculina }\end{array}$ & $\begin{array}{c}\text { Primacia del } \\
\text { del individuo }\end{array}$ \\
$\begin{array}{l}\text { Vínculo } \\
\text { mujer/trabajo }\end{array}$ & Trabajo & $\begin{array}{c}\text { Gratuidad e invisibilidad } \\
\text { del trabajo feminino }\end{array}$ & $\begin{array}{c}\text { Reconocimiento } \\
\text { sociăl }\end{array}$ \\
$\begin{array}{l}\text { Vínculo } \\
\text { mujer/patrimonio }\end{array}$ & $\begin{array}{c}\text { Rol en la conservación } \\
\text { del patrimonio }\end{array}$ & $\begin{array}{c}\text { La mujer al } \\
\text { servicio del patrimonio }\end{array}$ & $\begin{array}{c}\text { Ausencia del } \\
\text { patrimonio en el análisis }\end{array}$ \\
$\begin{array}{l}\text { Identidad de } \\
\text { las mujeres }\end{array}$ & $\begin{array}{c}\text { Complementaria } \\
\text { a la de los hombres }\end{array}$ & $\begin{array}{c}\text { Subordinada a la } \\
\text { de los hombres }\end{array}$ & Individualizada \\
\hline
\end{tabular}

La profesionalización de las mujeres fuera de la agricultura (trabajo asalariado) o en la explotación agrícola (actividades anejas) es una variable positiva de la emancipación de las mujeres fuera o dentro de la agricultura. La identidad de la mujer se inscribe cada vez más en un proyecto individual, que expresa la liberación de la mujer sujeto y protagonista de su destino.

Sin embargo, cualquiera que sea su situación profesional (asalariada exterior, agricultora, actividad aneja, etc.) las mujeres siguen enfrentándose, a nivel de su identidad, a fuertes contradicciones (Rieu, 1998) que alternan, según nuestra hipótesis, entre la imagen socialmente construida de la "nueva" agricultora (una mujer moderna, gestora, antiproductivista ${ }^{5}$ ) y la realidad cotidiana de su situación frente al vínculo entre familia y agricultura.

En efecto, en el caso de Francia, el trabajo simbólico de las organizaciones sindicales agrícolas, con la ayuda de los medios de comunicación, tiende a dar una nueva imagen de la agricultora, es decir de la agricultura. No es casualidad

\footnotetext{
5 En Francia, la socióloga Rose-Marie Lagrave describe de la manera siguiente lo que ella considera como la "nueva agricultora": "La diversificación de las producciones es su baza, su cordura financiera, su reflejo. Más críticas con la lógica productivista y el modelo empresarial, las agricultoras desarrollan actividades a su manera. Más sensibles con la calidad que con la cantidad, más razonables y razonadas en sus elecciones económicas y financieras, confian en la calidad de los productos y de la vida" (Lagrave, 1998: 25-26).
} 
si se califican las actividades de gestión, comercialización, turismo, etc., de actividades anejas, alternativas, que se valoran además como actividades femeninas. Según Rose-Marie Lagrave, las mujeres "aparecen ahora como los nuevos agentes de cambio rural, al convertir una fracción del sector agrícola en un sector de servicios".

Pero lo que esa nueva imagen social oculta es la desigualdad social y de sexo sobre la cual se construye: ese nuevo reparto de las actividades en la explotación reproduce en realidad las antiguas dicotomías tradicionales entre trabajo masculino y trabajo femenino, al inventar nuevas actividades femeninas, lo que conduce a una reproducción de las jerarquías: la mujer ya no es la ayudante de su marido en la explotación, sino que se convierte en su secretaria. En cuanto a las actividades turísticas, de servicios, etc. es un "trabajo aparte", el de la mujer; el "verdadero trabajo" (la agricultura) es el de los hombres. Ese nuevo reparto de las tareas nos muestra el difícil reconocimiento de las mujeres en la actividad agrícola. Tal y como lo subrayan algunos sociólogos, esas nuevas formas de profesionalización intra-agrícolas refuerzan la masculinización de la representación social del oficio de agricultor (Rieu, op. cit).

La evolución del sector agrícola desde hace 40 años y la evolución del punto de vista teórico sobre las mujeres en la agricultura nos muestran esa dificultad, que estaría vinculada a dos factores.

a) A la misma naturaleza del trabajo femenino (como producción social), cuyo efecto es hacer que la mecanización aleje a las mujeres de la esfera productiva. "En las sociedades modernas la división sexual del acceso a las técnicas está fundada en la definición de una relación diferente de los hombres y las mujeres con la naturaleza: sumisión para las mujeres, dominio para los hombres. Es en la definición de lo femenino como vinculado a la naturaleza en lo que se fundamenta la exclusión de las mujeres de la legitimidad técnica" (DauneRichard, 1998: 43). Es la relación con el trabajo y con la naturaleza la que funda la legitimidad del papel y del status de cada sexo en la agricultura familiar.

b) Al vínculo, cualquiera que sea la situación profesional de la mujer, entre agricultura y familia, que sigue rigiendo y organizando la reproducción del oficio de agricultor (al contrario de lo que la evolución del oficio ha podido suponer): "aunque las familias que viven en las explotaciones son cada vez menos agrícolas, no se puede concluir que la agricultura se esté haciendo cada vez menos familiar. En efecto, el funcionamiento familiar sigue rigiendo la transmisión de las explotaciones y permite comprender la diversidad de las renovaciones de los agricultores y el mantenimiento de la diversidad de las explotaciones" (Blanc, Brun, Delord y Lacombre, 1980: 323). Aunque la aparición de la familia conyugal permite suponer la desaparición de la familia patriarcal, no se puede concluir, por lo tanto, la desaparación de la familia patrimonial. La agricultura sigue siendo un oficio patrimonial (Jacques-Jouvenot, 1997) y el vínculo agricultura/familia, aun cuando ha cambiado, es todavía fundamental. 
Así, aunque la crítica de las feministas en contra de los etnólogos nos parece claramente legítima respecto a la cuestión de las relaciones de dominación, considero también que los análisis, sobre todo sociológicos, de los años 80-90 han olvidado un elemento sobre el cual los etnólogos habían construido sus investigaciones: la dimensión patrimonial de la agricultura como soporte de la reproducción del oficio de agricultor ${ }^{6}$. $\mathrm{Al}$ centrar exclusivamente su análisis en la cuestión de la profesionalización de las mujeres, los sociólogos han olvidado la cuestión del patrimonio ${ }^{7}$. Por lo tanto, es el patrimonio agrícola transmitido lo que constituye todavía la dimensión central del oficio y lo que sirve de vínculo entre familia y agricultura.

Nuestra investigación ${ }^{8}$ nos muestra que la cuestión del patrimonio no queda sin efectos sobre la trayectoria social y la identidad de las mujeres, tanto más si se integra una temporalidad larga en el análisis. Observamos así, por ejemplo, que si bien el trabajo asalariado exterior de las mujeres está efectivamente presente en numerosas explotaciones agrícolas, no es ni estable, ni definitivo. Al contrario, se observan reorientaciones profesionales de las mujeres que se instalan con su marido en la explotación después de varios años de actividad profesional no agrícola. Esas reorientaciones están vinculadas a dos elementos: - al número de hijos, lo cual tiene incidencia en la trayectoria de las mujeres (Blanc y Perrier-Cornet, 1989), y a la configuración estructural de la explotación agrícola. Las reorientaciones profesionales de las mujeres, lejos de estar vinculadas directamente a elecciones individuales, están determinadas por las condiciones objetivas de la explotación agrícolas es decir, vinculadas a la cuestión de cómo se transmite el patrimonio a los sucesores. Así, la creación de empresas de mujeres se parece en algunos casos a "instalaciones ficticias" que favorecen la instalación del hijo sucesor (Jacques-Jouvenot, 1987); por ejemplo, la creación de una "ferme-auberge" se inscribe así en los mecanismos de la sucesión-instalación (Capt, 1995).

${ }^{6}$ En 1987, las Editions du CNRS editaron un libro que trata directamente de la mujer y del patrimonio, pero en una perspectiva etno-histórica: Femmes et patrimoine dans les sociétés rurales de l'Europe Méditerranéenne, bajo la dirección de G. Ravis Giordani, Ed. CNRS, París.

${ }^{7}$ Entendemos por patrimonio el conjunto de las dimensiones siguientes: economía, bienes, trabajo agrícola/doméstico, dimensión simbólica y cultural, etc., es decir lo que circula entre generaciones.

${ }^{8}$ Realizada para la tesis doctoral, tesis comparativa bajo la dirección de Dominique JacquesJouvenot (Universidad de Franche-Comté, Francia) y la co-dirección de Julio Iglesias de Ussel (Universidad de Granada, España). La investigación ha sido realizada en una perspectiva cualitativa sobre la base de entrevistas minuciosas con $\mathbf{3 0}$ familias agrícolas de la provincia de Granada en España y 30 familias de la región de Franche-Comté en Francia. 
La literatura sociológica que ha abordado el tema de las mujeres en el medio agrícola fracasa cuando analiza, entre otras cuestiones, las contradicciones en su identidad, puesto que la mayoría de los estudios no toman en cuenta la dimensión central de la explotación agrícola, a saber: la reproducción del patrimonio. La mujeres, cualquiera que sea su situación profesional, no son extrañas a la transmisión del patrimonio y del oficio (aun cuando sus actividades estén cada vez más alejadas de las actividades de producción agraria). Hay que volver a estudiar su vínculo con la familia y volver a analizar la noción de familia agrícola integrando la dimensión patrimonial. Tener en cuenta esa dimensión supone reintegrar el parentesco en el análisis de la identidad social de las mujeres en el medio agrícola y considerar así la familia como "un sistema en el cual se traban relaciones conyugales e intergeneracionales cuyo objetivo fundamental es la transmisión del patrimonio" (Jacques-Jouvenot, 1998).

Sin negar la importancia fundamental de las cuestiones relativas al estatuto profesional de las mujeres en la agricultura, se trata de superar una perspectiva demasiado centrada en el tema de la profesionalización y en el análisis de las ideologías subyacentes a la legitimación de las relaciones de género (en términos de reparto de las tareas, lugar en la familia, etc.) para centrarnos en el vínculo esencial entre mujer y patrimonio: "Deseo de transmisión y afirmación de sí misma no son incompatibles" (Dechaux, 1997).

Al reintegrar el parentesco en el análisis, se invierte la perspectiva y se intenta comprender cómo la identidad social de la mujer, construida en las relaciones de parentesco, conduce a las mujeres a asumir un papel fundamental en la transmisión patrimonial, es decir, en la circulación de los patrimonios (este problema es objeto de nuestra tesis).

\section{BIBLIOGRAFÍA}

AUGUSTINS, G. (1989), Comment se perpétuer? Devenir des lignées et destins des patrimoines dans les paysanneries européennes, Société d'ethnologie, Nanterre, París.

BARTHEZ, A. (1982), Famille, travail et agriculture, Economica, París.

BERLAN, M. (1985), Hasard et nécessité: le travail des femmes dans les exploitations agricoles familiales, Aix-en-Provence, CERS.

(1987), “Le salaire de l'agricultrice : du vent?", Femmes Informations, Bulletin du CODIF, $\mathrm{n}^{\circ} 40$.

BERLAN, M. y R.M. PAINVIN (1980), Les conditions de vie et de travail des femmes dans les exploitations agricoles en France, INRA-ENSA, París.

BLANC, M. y P. PERRIER-CORNET (1989), Foncier, famille et développement des exploitations agricoles, INRA, París. 
RIS

REVISTA INTERNACIONAL DE SOCIOLOGÍA

No 29, Mayo - Agosto, 2001

BLANC, M., A. BRUN, B. DELORD y P. LACOMBE (1980), "L'agriculture française est-elle encore familiale?", in Les Agriculteurs et la Politique, bajo la dirección de Pierre Coulomb, Hélène Delorme, Bertrand Hervieu, Marcel Jollivet y Philippe Lacombe, Presses de la Fondation Nationale des Sciences Politiques, Paris.

BOURDIEU P. (1972), "Les stratégies matrimoniales dans le système de reproduction", Annales ESC, 4-5, pp. 1105-1129.

CAPT, D. (1995), "Les fermes-auberges dans le Massif Vosgien. Logiques économiques et processus de succession-installation", Economie et Sociologie Rurales, INRA, Dijon.

CANIOU J. (1980), L'enseignement agricole en France. De la fin du XIXème à nos jours, Thèse de doctorat de $3^{\text {ème }}$ cycle, Université René Descartes, París V.

DARRE, J-P. (1985), La parole et la technique, L'Harmattan, París.

DAUNE-RICHARD, A-M. (1998), "Femmes et travail, l'enjeu des qualifications", POUR, $\mathrm{n}^{\circ} 158, \mathrm{pp} .43$.

DECHAUX, J.H. (1997), Le souvenir des morts, PUF, París.

DELPHY, Ch. (1970), "L'ennemi principal”, Partisans, libération des femmes, année zéro, $n^{\circ} 54-55$.

FILIPPI, G. y Ch. NICOURT (1987), "Contribution à la définition d'un métier: agricultrice", Sociologie du Travail, $\mathrm{n}^{\circ}$ 4, pp. 478-479.

INSEE (1993), Les agriculteurs, portrait social, Coll, Contours et Caractères.

JACQUES-JOUVENOT, D. (1997), Choix du successeur et transmission patrimoniale, Ed. L'Harmattan, coll. Logiques Sociales, París.

KARNOOUH, Cl. (1980), "La démocratie impossible: parenté, politique et communauté", en Larmarche, Rogers, Karnoouh, Paysans, femmes et citoyens, lutte de pouvoir dans un village lorrain, Le Paradou, Actes Sud.

LAGRAVE, R.M. (1987), "L'agricultrice inclassable: les fonctions sociales du flou statistique”, en R.M. Lagrave (ed.), Celles de la terre, Edit. de l'EHESS, París.

(1998), “Les agricultrices: les oubliées de la recherche et du féminisme”, Lunes, nº 4 , juillet.

LAGRAVE, R.M. y J. CANIOU (1987), “Un statut mis à l'index”, en R.M. Lagrave (ed.), Celles de la terre. Agricultrice: l'invention politique d'un métier, Ed. de l'EHESS, París.

MENDRAS, H. (1984, ed. 1992), La fin des paysans, Coédition Babel-Actes Sud, París.

MICHEL, M. y J. VIARD (1977), La campagne inventée, Actes Sud, París.

PARSONS, T. y R. BALES (1955), Family, socialization and interaction process, Free Press, Glencoe. 
PERRIER-CORNET, P. y M. BLANC (1989), Foncier, famille et développement des exploitations agricole", INRA, París.

RAVIS GIORDANI, G. (ed.) (1987), Femmes et patrimoine dans les sociétés rurales de l'Europe Méditerranéenne, Ed. CNRS, París.

RIEU, A. (1998), “Agricultrices: itinéraires pour une reconnaissance”, Revue POUR, ${ }^{\circ} 158$, pp. 89.

ROGERS, S.C. (1980), "Les femmes et le pouvoir", en Lamarche, Rogers, Karnoouh, Paysans, femmes et citoyens, lutte de pouvoir dans un village lorrain, Le Paradou, Actes Sud.

SEGALEN, M. (1980), Mari et femme dans la société paysanne, París, Flammarion.

SINGLY de F. (1996), Le soi, le couple et la famille, Ed. Nathan, París.

VERDIER, Y. (1979), Façons de dire, façons de faire. La laveurse, la couturière, la cuisinière, Ed. Gallimard, París.

WHATMORE, S.J. (1989), "Ciclo vital o patriarcado? cambios en las divisiones del trabajo en la explotación agraria familiar por razón del sexo", Revista de Estudios Agro-sociales, $n^{\circ} 147$, Enero-Marzo.

ZONABEND, F. (1980), La mémoire longue, París, PUF.

JACQUES-JOUVENOT, D. (1998), “Parenté, travail des femmes et transmission des patrimoines", colloque SFR, Paris. 